\title{
TWO EMBEDDING THEOREMS FOR LATTICES
}

\author{
G. GRÄTZER AND C. R. PLATT ${ }^{1}$
}

\begin{abstract}
A lattice $L$ satisfies (SD $)$ if $a \wedge b=a \wedge c$ implies that $a \wedge b$ $=a \wedge(b \vee c)\left(\left(\mathrm{SD}_{\vee}\right)\right.$ is defined dually). THROREM. Every lattice can be embedded in the ideal lattice of a lattice satisfying $\left(\mathrm{SD}_{\wedge}\right)$ (respectively, $\left(\mathrm{SD}_{\vee}\right)$ ). Call a lattice $K$ transferable iff whenever $K$ can be embedded in the ideal lattice of a lattice $L$, then $K$ can be embedded in $L$. CorollarY. Every transferable lattice satisfies $\left(\mathrm{SD}_{\wedge}\right)$ and $\left(\mathrm{SD}_{\bigvee}\right)$.
\end{abstract}

1. Introduction. Two properties $(P)$ can be found in the literature for which the following embedding theorem holds:

Every lattice can be embedded in the ideal lattice of a lattice with property (P).

These are: (i) Sectional finiteness (that is, every principal ideal is finite) and (ii) property $(\mathrm{X})$ (that is, the lattice has no doubly reducible element).

References: (i) Whitman's embedding theorem [8] and the observation that the partition lattice is isomorphic with the ideal lattice of finite partitions (see, for instance, [6]); (ii) result announced in [4], proved in [5].

If for (P) the embedding theorem holds and in addition (P) is preserved under the formation of sublattices, then we can conclude that every transferable lattice satisfies $(\mathrm{P})$.

In this note we prove that $\left(\mathrm{SD}_{\wedge}\right)$ and $\left(\mathrm{SD}_{\vee}\right)$ are such properties. Observe that, although $\left(\mathrm{SD}_{\vee}\right)$ is the dual of $\left(\mathrm{SD}_{\wedge}\right)$, the two embedding theorems are not duals of each other.

2. Construction of a closure system. Let $\langle X, S\rangle$ be a closure system; that is, $X$ is a nonvoid set and $S$ is a set of subsets of $X$ closed under arbitrary intersections. For $Y \subseteq X$ let $\bar{Y}$ denote the smallest member of $S$ containing $X$. We define a closure system on $X \times Z$, where $Z$ is the set of integers:

Let $A \subseteq X \times Z$ be closed iff for $Y \subseteq X$ and $n \in Z, Y \times\{n\} \subseteq A$ implies that $\bar{Y} \times\{n-1\} \subseteq A$. For $Y \subseteq X \times Z$ let [Y] denote the closure of $Y$ in $X \times Z$. Call a subset of $X \times Z$ bounded iff $Y \subseteq X \times(n]$ for some $n \in Z$, where $(n]=\{i \mid i \in Z$ and $i \leqslant n\}$. Let $L(X)$ denote the set of all bounded closed subsets of $X \times Z$. Partially ordering $L(X)$ by set inclusion makes $L(X)$ a lattice.

Received by the editors May 18, 1977.

AMS (MOS) subject classifications (1970). Primary 06A20.

Key words and phrases. Lattice, semidistributive, embedding.

'The research of both authors was supported by the National Research Council of Canada. 
THEOREM 1. $L(X)$ is a lattice satisfying $\left(\mathrm{SD}_{\bigvee}\right)$.

Proof. We claim that for every $Y \in L(X)$, there is a (unique) $Y^{\prime} \subseteq Y$ with the following properties:

(i) $\left[Y^{\prime}\right]=Y$;

(ii) if $Y=[A]$, then $A \supseteq Y^{\prime}$.

Define, for each $i \in Z, Y_{i}=\{y \mid\langle y, i\rangle \in Y\}$. Thus, $Y=\cup\left(Y_{i} \times\{i\} \mid i \in\right.$ $Z$ ).

Define

$$
Y^{\prime}=\cup\left(\left(Y_{i}-\overline{Y_{i+1}}\right) \times\{i\} \mid i \in Z\right) .
$$

We claim that for all $i \in Z, Y_{i} \times\{i\} \subseteq\left[Y^{\prime}\right]$. Since $Y$ is bounded, $Y_{i}=\varnothing$ for sufficiently large $i$, and the inclusion is trivial. Suppose it has been established that $Y_{i+1} \times\{i+1\} \subseteq\left[Y^{\prime}\right]$. By definition of closure, $\overline{Y_{i+1}} \times\{i\}$ $\subseteq\left[Y^{\prime}\right]$. On the other hand, $\left(Y_{i}-\overline{Y_{i+1}}\right) \times\{i\} \subseteq Y^{\prime} \subseteq\left[Y^{\prime}\right]$ by definition. This verifies (i).

To verify (ii), let $Y=[A]$ and $\langle x, j\rangle \in Y^{\prime}$. Then $x \notin \overline{Y_{j+1}}$, and it follows that $Y-\{\langle x, j\rangle\}$ is closed. If $\langle x, j\rangle \notin A$, then $Y-\{\langle x, j\rangle\}$ would be a closed set containing $A$, hence would contain $[A]=Y$, a contradiction. This proves (ii).

Observe that in $L(X), A \vee B=[A \cup B]$ and $A \wedge B=A \cap B$. To verify $\left(\mathrm{SD}_{\vee}\right)$ in $L(X)$, let $A \vee B=A \vee C=D$. $\left[A^{\prime} \cup B^{\prime}\right]=\left[A^{\prime} \cup C^{\prime}\right]=D$ by (i) and the description of $\vee$ in $L(X)$. Then $D^{\prime} \subseteq A^{\prime} \cup B^{\prime}$ and $D^{\prime} \subseteq A^{\prime} \cup C^{\prime}$, hence $D^{\prime} \subseteq A^{\prime} \cup\left(B^{\prime} \cap C^{\prime}\right)$. Consequently,

$$
D=\left[D^{\prime}\right] \subseteq\left[A^{\prime}\right] \vee\left[B^{\prime} \cap C^{\prime}\right] \subseteq A \vee(B \wedge C) \subseteq D,
$$

hence $D=A \vee(B \wedge C)$. This completes the proof of Theorem 1 .

3. Embedding theorem for $\left(\mathrm{SD}_{\bigvee}\right)$. With a lattice $K$ we associate a closure system $K_{I}=\left\langle K, S_{I}\right\rangle$, the nonvoid members of $S_{I}$ being exactly the ideals of $K$.

THEOREM 2. Every lattice $K$ can be embedded in the ideal lattice of $L\left(K_{I}\right)$.

By Theorem 1 we then have the

COROLlaRY. Every lattice can be embedded in the ideal lattice of a lattice satisfying $\left(\mathrm{SD}_{\bigvee}\right)$.

For finite lattices, this Corollary was proved in [3].

Proof of Theorem 2. For $X \in L\left(K_{I}\right)$, let $\pi_{K}(X)$ be the projection of $X$ into $K$ and $\pi_{Z}(X)$ the projection of $X$ into $Z$. (Recall that $X \subseteq K \times Z$.) We define a map $\varphi$ by

$$
\varphi(x)=\left\{X \mid X \in L\left(K_{I}\right) \text { and } \pi_{K}(X) \subseteq(x]\right\}
$$

for $x \in K$. In words, $\varphi(x)$ consists of all bounded closed sets containing only elements whose $K$-component is $\leqslant x$. For $x \in K, \varphi(x)$ is obviously an ideal of $L\left(K_{I}\right)$.

It is clear that $\varphi$ is one-to-one and meet-preserving. To show that $\varphi$ is 
join-preserving it is sufficient to verify that $\varphi(x \vee y) \subseteq \varphi(x) \vee \varphi(y)$. Indeed, let $A \in \varphi(x \vee y)$. Since $A$ is bounded, $A \subseteq X \times(i]$ for some $i \in Z$. Then ${ }^{2}$ $A \subseteq[\langle x \vee y, i+1\rangle]$. Also, $[\langle x, i+2\rangle] \in \varphi(x),[\langle y, i+2\rangle] \in \varphi(y)$, therefore,

$$
A \subseteq[\langle x \vee y, i+1\rangle] \subseteq[\langle x, i+2\rangle] \vee[\langle y, i+2\rangle] \in \varphi(x) \vee \varphi(y),
$$

and so $A \in \varphi(x) \vee \varphi(y)$, completing the proof.

4. Embedding theorem for $\left(\mathrm{SD}_{\wedge}\right)$. Now we associate with a lattice $K$ a closure system $K_{D}=\left\langle D, S_{D}\right\rangle$, the nonvoid members of $S_{D}$ being exactly the dual ideals of $K$.

THEOREM 3. Every lattice $K$ can be dually embedded in the dual ideal lattice of $L\left(K_{D}\right)$.

(The dual ideal lattice is ordered by reverse set inclusion.)

Again, by Theorem $1, L\left(K_{D}\right)$ satisfies $\left(\mathrm{SD}_{\vee}\right)$. Let $K^{d}$ denote the dual of $K$. Then Theorem 3 yields that $K$ can be embedded in the ideal lattice of $L\left(K_{D}\right)^{d}$. Thus we obtain the following

COROLlaRY. Every lattice can be embedded in the ideal lattice of a lattice satisfying $\left(\mathrm{SD}_{\wedge}\right)$.

For finite lattices, this Corollary was proved in [3].

Proof of TheOREM 3. We define a map $\psi$ by

$$
\psi(x)=\left\{X \mid X \in L\left(K_{D}\right) \text { and } x \in \pi_{K}(X)\right\}
$$

for $x \in K$. It is easily seen that for $x \in K, \psi(x)$ is a dual ideal of $L\left(K_{D}\right)$. It is clear that $\psi$ is one-to-one, and since $x \wedge y \in \pi_{K}(X)$ iff $x$ and $y$ are in $\pi_{K}(X)$ $\left(x, y \in K, X \in L\left(K_{D}\right)\right){ }^{3}$ we get that $\psi(x \wedge y)=\psi(x) \cap \psi(y)=\psi(x) \bigvee$ $\psi(y)$. To show that $\psi(x \vee y)=\psi(x) \wedge \psi(y)$, that is, $\psi(x \vee y)$ is the dual ideal generated by the dual ideals $\psi(x)$ and $\psi(y)$, it is sufficient to show that for every $A \in \psi(x \vee y)$ there exist $B \in \psi(x)$ and $C \in \psi(y)$ satisfying $A \supseteq$ $B \cap C$. To this end, let $A \in \psi(x \vee y)$, that is, $\langle x \vee y, i\rangle \in A$ for some $i \in Z$. Set $B=[\langle x, i\rangle]$ and $C=[\langle y, i\rangle]$. Then $B \in \psi(x)$ and $C \in \psi(y)$. Let $\langle z, j\rangle \in B \cap C$. Then $z \geqslant x \vee y$ and $j \leqslant i$. If $j\langle i$, then $\langle z, j\rangle \in[\langle x \vee$ $y, i\rangle] \subseteq A$. If $j=i$, then we must have $x=y=z$ and so again $\langle z, j\rangle \in A$. Thus $B \cap C \subseteq A$, completing the proof that $\psi$ is a dual embedding and thus finishing the proof of the theorem.

\section{REFERENCES}

1. R. Antonius and I. Rival, A note on Whitman's property for free lattices, Algebra Universalis 4 (1974), 271-272.

2. K. A. Baker and A. W. Hales, From a lattice to its ideal lattice, Algebra Universalis 4(1974), 250-258.

\footnotetext{
${ }^{2}$ For $a \in X \times Z$, let $[a]$ denote $[\{a\}]$.

${ }^{3}$ To prove this observe that if $\langle x, i\rangle$ and $\langle y, j\rangle$ are in $X$, then $\langle x \wedge y, k-1\rangle \in X$ where $k=i \wedge j$. This is the reason it is necessary to use $Z$ rather than $\omega$ in the construction of $L\left(K_{D}\right)$. For Theorems 1 and $2, Z$ could be replaced by the positive integers.
} 
3. H. S. Gaskill, G. Grätzer and C. R. Platt, Sharply transferable lattices, Canad. J. Math. 27 (1975), 1247-1262.

4. G. Grätzer, Universal algebra, Trends in Lattice Theory, Van Nostrand, New York, 1970, pp. 173-215.

5. __ A property of transferable lattices, Proc. Amer. Math. Soc. 43 (1974), 269-271.

6. General lattice theory, Birkhäuser, Basel, 1978.

7. Ph. M. Whitman, Free lattices, Ann. of Math. (2) 42 (1941), 325-330.

8. __Lattices, equivalence relations, and subgroups, Bull. Amer. Math. Soc. 52 (1940), $507-522$.

Dipartaient of Mathimatics, University of Manttoba, Winnipeg, Manttoba, Canada 